\title{
Continuing professional development: Western Australian radiographers' opinions and attitudes
}

\begin{abstract}
Purpose: The Australian radiography profession is currently moving towards compulsory continuing professional development (CPD) participation. An understanding of radiographer's attitudes and opinions towards CPD will assist in providing a CPD programme that meets the needs of radiographers. The aim of this study was to determine Western Australian radiographers' attitudes and opinions towards CPD and the programme that is currently being run by the Australian Institute of Radiography. Methods: Two-hundred and seventy-seven questionnaires about CPD were distributed to metropolitan clinical centres across Perth and 15 rural centres in Western Australia. Results: A total of 192 questionnaires were returned. A response rate of $64 \%$ was achieved for the metropolitan area (178/277). Among all respondents, $88 \%$ were currently engaged in CPD and the group of age $50+$ had the highest percentage (78.1\%) of respondents who believed CPD to be very important. Updating professional knowledge (79\%) and maintaining professional competence (75\%) were the most popular reasons for participating in CPD. Participants reported that time constraints and workload are barriers to CPD participation. Employer initiated CPD was most favoured; with in-house education and workplace seminars as suggested methods to increase CPD participation. Conclusion: This study demonstrated that decreased participation rates in CPD were mainly due to time constraints and workloads. Participation in CPD programmes is likely to be increased with more in-house education and workplace seminars. Changes need to be made to the current CPD programme to improve its accessibility and usability
\end{abstract}

Keywords: Continuing professional development, CPD, continuing education, radiography, role development.

\section{Introduction and background}

Continuing professional development $(\mathrm{CPD})$ is defined by the Australian Institute of Radiography (AIR) as "ongoing maintenance and growth of professional excellence through participation in life long activities, which are planned and implemented to achieve this for the benefit of participants, patients and the

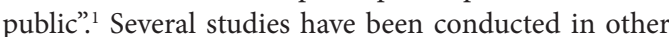
countries regarding the importance of CPD and the attitudes that radiographers have towards the particular systems. A study conducted in Europe by Marshall, et al. ${ }^{2}$ established that $54 \%$ of the radiographers who participated in the study believed that CPD was very important. According to Gould, et al., ${ }^{3}$ CPD plays an important role in the improvement of job satisfaction by providing the opportunity to improve within one's field, which therefore results in increased confidence and job security. Furthermore, CPD may be effective in encouraging radiographers to stay within the profession.

Henwood, et al. ${ }^{4}$ conducted a two-phase study to determine the attitudes of radiographers towards a mandatory CPD policy being enforced in New Zealand and the United Kingdom. The main areas of concern were short staffing, timing, management/employer attitudes, salary issues and remoteness/access. Many participants indicated that employers did not provide enough support and encouragement to their staff.

Until recently there has been a lack of published evidence regarding Australian radiographers' opinions about the AIR CPD programme. Lee, et al. ${ }^{5}$ recently conducted a study with New South Wales radiographers about their perceptions of the current AIR CPD project. One hundred and eighty-eight radiographers participated (response rate $=47 \%$ ). Constraints limiting participation in the CPD programme included lack of time, lack of funding, lack of support, lack of access and other commitments. Of participants, $80 \%$ felt that the activities currently available were adequate and 63\% of participants thought that participation in the $\mathrm{CPD}$ programme should remain mandatory for members of the AIR. Sim ${ }^{6}$ highlighted that the AIR needs to meet the following challenges:

- Modify the CPD programme to meet the varying aspirations of medical radiation practitioners

- Foster greater collaboration between institutions

- Work together to develop a holistic CPD model.

The aim of this study was to explore Western Australian radiographers' attitudes and opinions towards mandatory $\mathrm{CPD}$, the benefits they feel $\mathrm{CPD}$ provides and the areas of interest that will enhance CPD learning and participation. Secondary aims of this study were to determine:

- If time since graduation affects radiographers' attitudes towards CPD

- The CPD activities that radiographers believe enhance effective learning

- Barriers and limitations that prevent radiographers from participating in CPD activities.

This project was conducted as a fourth year research project as part of the Degree of Medical Imaging Science
H Sholer ${ }^{1}$

BSc (Medical Imaging Science) (Hons) $)^{1}$

S Tonkin ${ }^{1}$

BSc (Medical Imaging Science) (Hons)

K F Lau ${ }^{1}$

BSc (Medical Imaging Science) (Hons)

C Law

BSc (Medical Imaging Science) (Hons)

R Rahman ${ }^{1}$

BSc (Medical Imaging Science) (Hons) ${ }^{1}$

GKB Halkett ${ }^{1,2}$

PhD, FIR, BMedRad(Hons)

${ }^{1}$ Discipline of Medical Imaging Science

Faculty of Science and Engineering, Curtin University,

Perth, WA 6845, Australia.

${ }^{2}$ WA Centre for Cancer and Palliative Care and Curtin Health Innovation Research Institute

Curtin University

Perth, WA 6845, Australia.

Correspondence to

G.Halkett@curtin.edu.au 
Table 1: Participant demographics, $n=192$.

\begin{tabular}{lcc} 
& Number & Percentage \\
\hline Age & & \\
$21-25$ & 40 & $20.8 \%$ \\
$26-30$ & 39 & $20.3 \%$ \\
$31-40$ & 42 & $21.9 \%$ \\
$41-50$ & 39 & $20.3 \%$ \\
$50+$ & 32 & $16.7 \%$ \\
Years of experience & & \\
Completing professional development & 3 & $1.6 \%$ \\
year & 56 & $29.2 \%$ \\
$1-5$ years & 43 & $22.4 \%$ \\
$6-10$ years & 28 & $14.6 \%$ \\
$11-20$ years & 62 & $32.3 \%$ \\
$20+$ years & & \\
Position & 145 & $75.5 \%$ \\
Full time & 42 & $21.9 \%$ \\
Part time & 5 & $2.6 \%$ \\
Casual & & \\
Type of practice & 103 & $53.6 \%$ \\
Public & 89 & $46.4 \%$ \\
Private & & \\
Area of practice & 178 & $7.3 \%$ \\
Metropolitan & 14 & \\
Rural & & \\
\hline
\end{tabular}

at Curtin University in 2009. This research complements the recent study on CPD attitudes in NSW by Lee, et al. ${ }^{5}$

At the time of conducting the study the Medical Radiation Technologists Registration Board of Western Australia's (MRTRBWA) was planning to introduce mandatory CPD from 1st July 2009. The MRTRBWA guidelines indicated that Western Australia radiographers would be required to provide evidence of at least 10 hours of CPD activities per year in order to renew their registration from 1st July 2010.

\section{Methods}

Ethics approval was gained from the Curtin University Human Research Ethics Committee (HREC number: ESC-06-2009, approval date: June 2009).

\section{Materials}

The content of the questionnaire was informed by previous studies on CPD. ${ }^{2-4,7-10}$ The questionnaire was divided into four parts:

- The demographics of the participants

- Opinions about participating in CPD

- For those who currently undertake CPD, determining the type of $\mathrm{CPD}$ activities they undertake and their opinions about how these activities have enhanced their knowledge and work skills

- Impediments preventing participation in CPD.

Participants

Participant inclusion criteria was any radiographer currently registered
Table 2: Employment status versus age of participants.

\begin{tabular}{lccccc} 
Employment status & \multicolumn{5}{c}{ Age } \\
\hline & $\mathbf{2 1 - 2 5}$ & $\mathbf{2 6 - 3 0}$ & $\mathbf{3 1 - 4 0}$ & $\mathbf{4 1 - 5 0}$ & $\mathbf{5 0 +}$ \\
Full time & $100 \%$ & $95 \%$ & $79 \%$ & $54 \%$ & $44 \%$ \\
Part time & $0 \%$ & $3 \%$ & $19 \%$ & $38 \%$ & $56 \%$ \\
Casual & $0 \%$ & $3 \%$ & $2 \%$ & $8 \%$ & $0 \%$ \\
\hline
\end{tabular}

with the MRTRBWA. Exclusion criteria included those who are currently employed on working visas and those who do not administer ionising radiation, such as sonographers and Magnetic Resonance Imaging (MRI) technologists.

\section{Sample size}

As of December 2008 there were 811 Medical Imaging Technologists registered with the MRTBWA. ${ }^{11}$ Sample size calculation software (Raosoft, Seattle, WA, USA) was used to calculate the sample size required to obtain a representative sample of radiographers. To achieve a confidence level of $95 \%$, with a $10 \%$ margin of error for questions with yes/no responses, a sample of at least 86 participants was required..$^{12}$ It was anticipated that it would be necessary to distribute 300 questionnaires to ensure this sample size was achieved.

\section{Data collection}

A list of centres was obtained from the MRTBWA and used to ensure a broad spectrum of clinical centres were included. This list included centres from both metropolitan and rural areas of Western Australia.

An information letter was attached to the self-completed questionnaire detailing the relevant criteria for participation in the study. The members of the research team were responsible for distributing the questionnaires to 28 metropolitan centres. The research team provided the questionnaires to an allocated representative within the centres and followed up one week later to obtain feedback and organise a suitable time for retrieval of the completed questionnaires. This permitted a turnaround time of two weeks, which allowed for prompt processing of the results. This method of recruitment ensured anonymity of individual participants.

Fifteen rural centres were approached by email. Questionnaires with the relevant information letter were attached to an email requesting their participation, with completed questionnaires required within a two-week time frame. All completed questionnaires were to be sent to the Department of Imaging and Applied Physics at Curtin University, to ensure anonymity of the participants. A reminder email was sent a week later thanking those who had participated and reminding those who had not of the required return date.

Data analysis

The data were entered and managed with the statistical software Statistical Package for the Social Sciences (SPSS) version 17 (College Station, TX, USA). This programme was used to calculate descriptive statistics including percentages and frequencies, to describe the sample and its characteristics. Comparisons were made between sub groups within the sample using the chi-square test for categorical variables.

\section{Results}

In the metropolitan area, 277 surveys were distributed and 178 were returned, constituting a response rate of $64 \%$. Of the 15 rural centres 


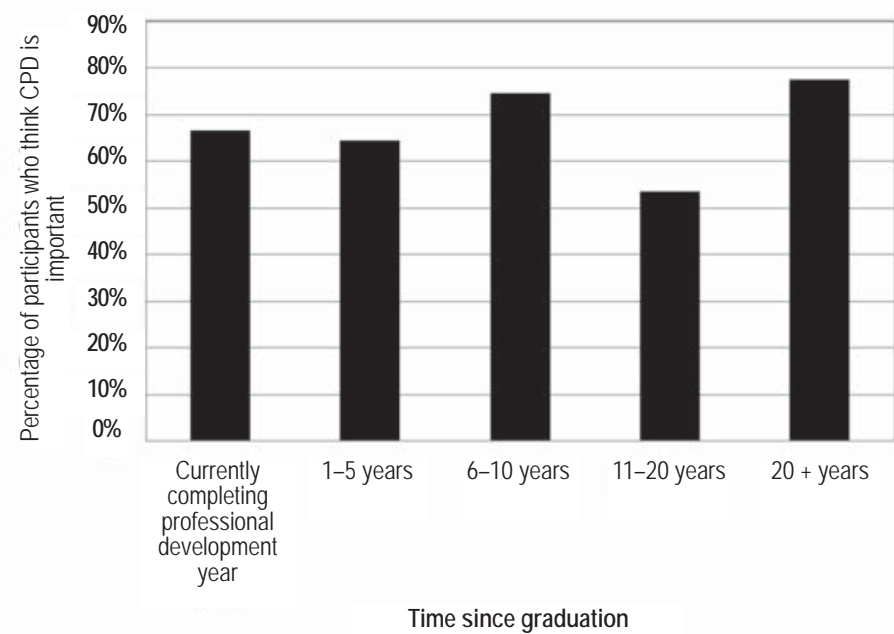

Figure 1: Participants' opinions regarding the importance of Continuing Professional Development (CPD) in order to remain professionally competent.

approached, nine centres returned 14 completed questionnaires. It is not known how many radiographers work at each of these rural centres, so a response rate cannot be calculated.

The demographic data of participants is presented in Tables 1 and 2. Table 1 describes the age, years of experience as a radiographer, the type and area of practice worked in and the position held at the particular practice.

Table 2 demonstrates the distribution of participation in terms of age and employment status.

\section{Importance of CPD to remain professionally competent}

Figure 1 demonstrates the percentage of participants who believed that $\mathrm{CPD}$ was required to remain professionally competent.

Of participants, $80 \%$ believed CPD should be structured by their employer, with $84 \%$ indicating that their employer assisted them in obtaining CPD and $89 \%$ were aware that CPD had become mandatory in Western Australia as of 1st July 2009, with 58\% believing CPD should be mandatory. Of participants, $69 \%$ believed that CPD was required to remain professionally competent. Chi-square tests demonstrated that participants who believed that $\mathrm{CPD}$ was required to remain professionally competent also believed that CPD should be mandatory $(0.05<P<0.02)$.

\section{Reasons for completing CPD}

Radiographers who were engaged in CPD $(n=169)$ were asked to describe the reasons why they completed CPD. The options provided were:

- To increase my professional knowledge

- To fulfil statutory requirements in order to register

- Other, please specify.

Participants were able to select more than one response. Of participants, $70 \%$ indicated that they participated in CPD to fulfil statutory requirements in order to register. Of participants $55 \%$ also indicated that they participated in CPD to increase their professional knowledge.

Popular CPD activities

Employer initiated information sessions and student supervision were
Table 3: Continuing Professional Development activities undertaken by participants and their opinions about whether these activities are beneficial.

\begin{tabular}{lcc} 
Activity & $\begin{array}{c}\text { Participate } \\
\text { in the activity }\end{array}$ & $\begin{array}{c}\text { Believe it to } \\
\text { be beneficial }\end{array}$ \\
\hline $\begin{array}{l}\text { Reading scholarly literature such as } \\
\text { journals }\end{array}$ & $50.5 \%$ & $75.3 \%$ \\
Completion of crosswords & $25.5 \%$ & $44.9 \%$ \\
AIR run courses or seminars & $40.1 \%$ & $90.9 \%$ \\
Employer initiated information session & $71.9 \%$ & $83.3 \%$ \\
Cannulation course & $41.7 \%$ & $83.8 \%$ \\
Courses on PACS applications & $25.5 \%$ & $73.5 \%$ \\
Student supervision & $71.4 \%$ & $65.0 \%$ \\
Further graduate studies & $15.6 \%$ & $70.0 \%$ \\
Journal clubs & $2.1 \%$ & $50.0 \%$ \\
\hline
\end{tabular}

Australian Institute of Radiography (AIR), Picture Archiving and Communication System (PACS)

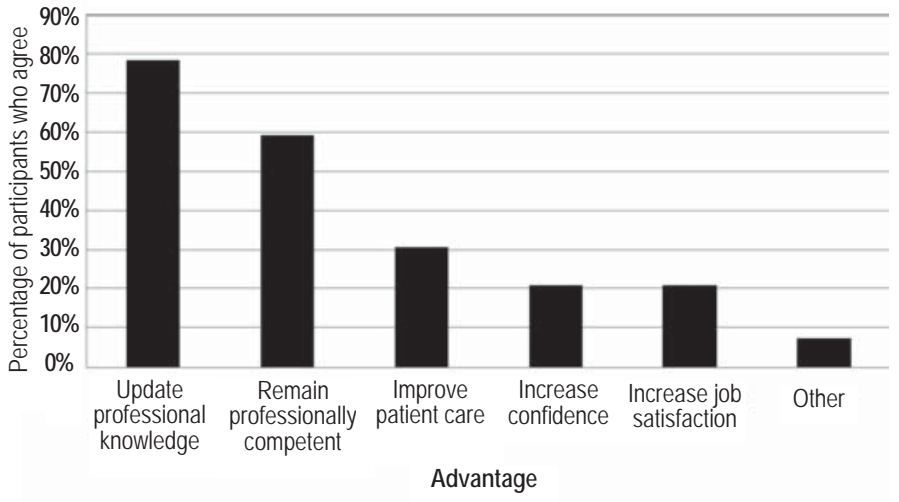

Figure 2: Participants' opinions on advantages of participating in Continuing Professional Development.

the most popular CPD activities at $72 \%$ and $71 \%$ respectively. Reading scholarly literature $(51 \%)$ and attending AIR run courses and seminars (40\%) were also common activities. Completion of crosswords and courses in Picture Archiving and Communication System (PACS) applications, at $26 \%$ each, were the other most noteworthy activities recorded by the study's participants.

Table 3 demonstrates the activities that radiographers participated in, and whether they believed that these activities were beneficial.

\section{Perceived advantages of CPD}

Figure 2 demonstrates the responses of all participants with regards to the advantages of $\mathrm{CPD}$. These results suggest that the respondents believed CPD helped to maintain professional competence and also updated professional knowledge more than other reasons such as improving patient care. Almost $60 \%$ of all participants agreed that CPD updated professional knowledge and also helped maintain professional competence. Approximately $20 \%$ believed that CPD increased confidence and/or job satisfaction.

Participants were also asked whether CPD enhanced their skills. Table 4 summarises how CPD had enhanced their skills. 
Table 4: Ways that Continuing Professional Development has enhanced participants' skills.

\begin{tabular}{lc} 
Skill/knowledge enhanced & Percentage \\
\hline Communication skills & $14.1 \%$ \\
Understanding of current technology & $64.4 \%$ \\
Awareness of current legal requirements & $24.5 \%$ \\
Basic diagnostic skills & $38 \%$
\end{tabular}

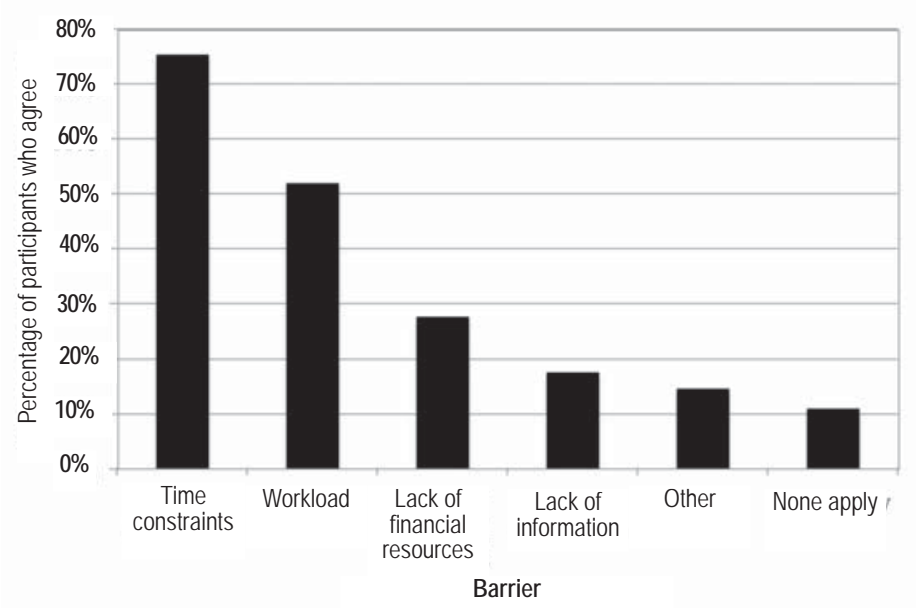

Figure 3: Barriers that limit participation in Continuing Professional Development.

\section{Barriers that limit CPD participation}

Participants identified barriers that limited their participation in CPD (Figure 3).

Of radiographers surveyed, $76 \%$ found time to be a major barrier, and $52 \%$ thought that their workload made it difficult to engage in CPD activities. Some $49 \%$ of radiographers reported that they had no time due to after hours commitments and $39 \%$ believed that employers should support CPD with time and remuneration allowances.

Participants also identified methods that might increase their participation (Figure 4).

Of participants, $84 \%$ believed that workplace organised seminars would help to increase participation in CPD and $82 \%$ believed in-house education would encourage participation. Approximately 50\% also indicated that monetary subsidies and time off from work would also encourage CPD participation.

\section{Additional education topics}

Table 5 demonstrates participants' preferences for additional education topics to be provided.

\section{Differences between public and private sector}

Among radiographers working privately, 92\% reported that they received assistance from their employer compared to a much lower $77 \%$ of radiographers working in the public sector. This is statistically significant with a chi-square value of $8.40(0.01<P<0.001, \mathrm{DF}=1)$. Of radiographers working in the private sector $52 \%$ had someone in their department who was responsible for training, compared to only $40 \%$ of radiographers in the public sector. Furthermore, $74 \%$ of private radiographers reported that CPD

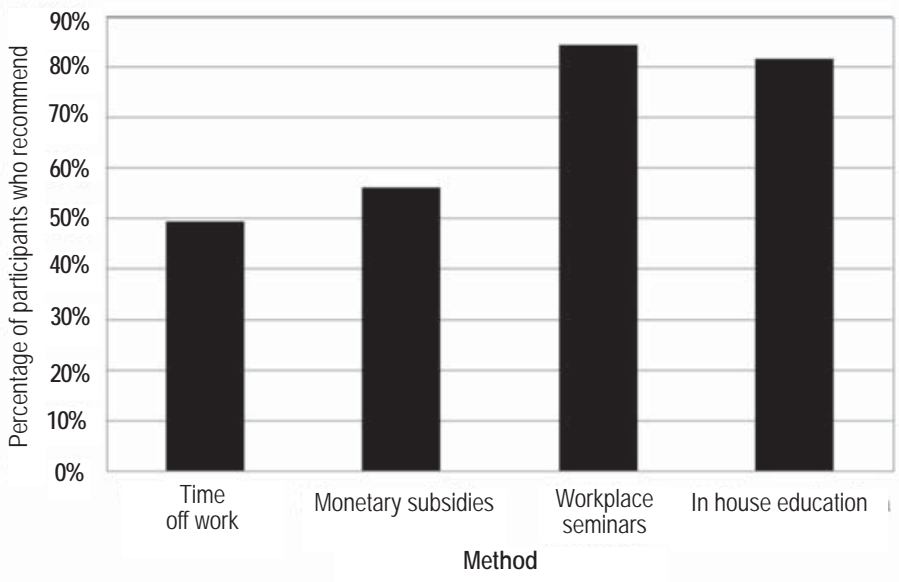

Figure 4: Participants' opinions on methods to increase participation in Continuing Professional Development.

Table 5: Additional continuing professional development education topics that participants would like to see provided.

\begin{tabular}{lc} 
Topics & Percentage \\
\hline Specialisation (CT, MRI) & $76 \%$ \\
Advances in technology such as PACS & $58 \%$ \\
Knowledge of current law & $28 \%$ \\
Communication skills & $15 \%$
\end{tabular}

Computer Tomography (CT), Magnetic Resonance Imaging (MRI),

Picture Archiving and Communication System (PACS)

was important to remain professional competent, compared to only $65 \%$ of public radiographers. At the time of the survey, $98 \%$ of radiographers working in the private sector were engaged in CPD, whereas only $80 \%$ of radiographers in the public sector were participating in CPD.

\section{Discussion}

In 2004, Field ${ }^{13}$ summarised the debate as to whether CPD should be voluntary or mandatory, concluding that $\mathrm{CPD}$ should remain voluntary until evidence demonstrates that mandatory CPD improves professional performance. This study contributes to our understanding of radiographers' current attitudes towards CPD. In particular, it provides an understanding of Western Australian radiographers' attitudes and opinions towards mandatory CPD, the benefits they feel CPD provides and the areas of interest that will enhance CPD learning and participation.

Overall, $58 \%$ of participants in this study thought CPD should be mandatory. This is very similar to the percentages reported by Henwood, et $a l .{ }^{4}$ and Lee, et al. ${ }^{5}$. Participants in this study commonly reported that they participated in CPD not only to fulfil statutory requirements (70\%), but also to increase professional knowledge (55\%). This finding demonstrates that many radiographers recognise the importance of continuing their professional development post graduation and that the CPD programme is being used to meet its designed purpose. Further research after the mandatory CPD programme has been implemented for several years would be beneficial to determine whether participants are still participating to increase their professional knowledge and whether the programme is meeting their current needs. Marshall, et al. ${ }^{7}$ reported that recently qualified radiographers believed participation in CPD was more valuable 
than radiographers who had been qualified for longer. The data collected in the current study indicates that this was not reflective of the majority of participants. In the current study 59\% of participants believed CPD was required to remain professionally competent with $49 \%$ of these participants being six plus years post graduation. Many of these radiographers who are six plus years post graduation may hold managerial positions or be senior radiographers within their department and could play an influential role in increasing CPD participation by setting an example and providing leadership. It may be beneficial for the AIR to work with these radiographers to create suitable CPD programmes and increase participation.

Participants in this study were asked to identify the types of activities that they were undertaking to obtain CPD points. Employer initiated information sessions were the most popular with $72 \%$ of the participants indicating they partook in these sessions. Of participants in this study $71 \%$ had actively undertaken supervision of these students. This form of CPD allows the radiographers to revisit their basic skills and reflect on enhancements they have made to their own practices. To keep updated with the latest research and developments in their field, 51\% read scholarly literature as a CPD activity. Computed tomography radiographers in private practice are at times required to cannulise their patients prior to contrast enhancement studies. The AIR regularly run courses and seminars and advertise their availability locally, on their website ${ }^{14}$ and in Spectrum. Of participants surveyed $40 \%$ indicated that they had attended these events. Further advertisement and availability of these seminars is likely to increase participation in the future.

Participants in the current study were also asked to identify barriers that prevented their participation in CPD. Time constraints (76\%) and workload (52\%) were the main factors identified. Henwood, et al. ${ }^{4}$ similarly reported that lack of time, employer attitudes and access were the main deterrents to participating in CPD. Lee, et al. ${ }^{5}$ reported that lack of time, lack of access and lack of funding were common barriers to participation in CPD. Common barriers to CPD participation identified by rural radiographers in the current study were distance and expenses associated with CPD activities. Marshall, et al. ${ }^{2}$ found that providing more internet based activities would improve participation. This is relevant to the current study because time and travel could be less of an issue for radiographers if they were able to partake in CPD via the internet. The AIR and individual centres will need to consider these barriers when organising CPD activities for radiographers in metropolitan and rural areas throughout Australia.

It is encouraging to report that $77 \%$ of public and $92 \%$ of private sector workers indicated that their employers supported them in obtaining CPD. Munro ${ }^{10}$ evaluated the CPD framework for nursing in the UK and developed some recommendations on how CPD outcomes could be improved. Her recommendations were that CPD should be adapted so that learning may be conducted through workplace learning rather than unstructured learning without purpose, employers need to be active in the learning criteria of their staff and all learning should be certificated and accredited to increase employee motivation. The results of this study reflect that a similar concept may be adapted and/or further encouraged within the Australian CPD system.

This study achieved a high response rate of $64 \%$ in the metropolitan area. However, there were several limitations to this study that should be noted. Rural participation in this study was limited due to time constraints and not being able to approach these departments personally. The method of distributing the survey in metropolitan areas was limited by the fact that team members chose which departments to approach based on their clinical placements. However, this method also increased the number of responses received. The research team were unable to identify individual participants within the departments selected. The timing of the questionnaire distribution did not allow determination of the CPD participation rate prior to mandatory implementation. However, this study does provide an indication of CPD participation at the time of the surveys being completed (June 2009). Finally, this study was Western Australia specific and focused on diagnostic radiographers' opinions and attitudes only.

\section{Conclusion}

This study has provided an insight into Western Australian radiographers' perceptions of CPD. Radiographers identified that time constraints and workload prevented them from participating in CPD. Active participation is likely to be increased with the provision of more in-house education and workplace seminars. Improvements need to be made to the current CPD programme to improve its accessibility and usability.

Since the completion of this study, research on CPD participation has also been completed in other states in Australia. ${ }^{5}$ It is proposed that in 2012 all state registration bodies will amalgamate into one national body that is managed by a central institution such as the AIR. ${ }^{15}$ Additional research including both radiographers and radiation therapists nationally will assist in improving CPD participation and is likely to further enhance the current CPD programme.

\section{Acknowledgements}

This project was a fourth year student project conducted at Curtin University. All students contributed equally to the project. Dr Georgia Halkett provided supervision throughout the project and assisted in the final preparation of this manuscript.

\section{References}

1 Australian Institute of Radiography. Guide to AIR continuing professional development triennial program 2007 Available online at: http://www.air.asn.au// files/08_CPD/0316_CPD_Guide1.pdf [verified 10th Feb 2009].

2 Marshall G, Punys V, Sykes A. The continuous professional development (CPD) requirements of radiographers in Europe: An initial survey. Radiography 2008; 14: 332-42.

3 Gould D, Kelly D, Goldstone D, Maidwell A. The changing training needs of clinical nurse managers: exploring issues for continuing professional development. J Adv Nurs 2001; 34: 7-17.

4 Henwood SM, Yielder J, Flinton D. Radiographers attitudes to mandatory CPD: a comparative study in the United Kingdom and New Zealand. Radiography 2004; 10: 251-8.

5 Lee SHE, Reed W, Poulos A. Continuing Professional Development: the perceptions of radiographers in New South Wales. The Radiographer 2010; 57: 33-9.

6 Sim J. Continuing Professional Development: are we at the crossroads? The Radiographer 2010; 57: 5-6.

7 Henwood SM, Taket A. A process model in continuing professional development: Exploring diagnostic radiographers' views. Radiography 2008; 14: 206-15.

8 Henwood SM, Huggett SM. Radiographic CPD requirements-a regional study. Radiography 1999; 5: 3-10.

9 Castle A, Adrian-Harris D, Holloway DG, Race AJ. Continuing professional development for radiographers. Radiography 1997; 3: 253-63.

10 Munro KM. Continuing professional development and the charity paradigm: interrelated individual, collective and organisational issues about continuing professional development. Nurse Educ Today 2008; 28: 953-61.

11 The Medical Radiation Technologists Registration Board of Western Australia. MRTBWA Newsletter. 2008. Available online at: http://www.mrtboard.wa.gov.au/ 
MRT\%20Newsletter\%20Edition\%202.pdf [verified 26th Mar 2009].

12 Raosoft. Sample size calculator 2004. Available online at: http://www.raosoft.com/ samplesize.html [verified 23rd Mar 2009].

13 Field N. Mandatory Continuing Professional Education: Do we need it? The Radiographer 2004; 51: 5-9.

14 Australian Institute of Radiography. AIR event calendar. 2009. Available online at http://www.air.asn.au/events.php [verified 12th Nov 2009].
15 The National Steering Committee for Medical Radiation Practitioners. Towards a safe medical radiation workforce. 2008 Available online at: http://www.mrtboard. wa.gov.au/NationalregistrationsubmissionMRT1.pdf [verified 30th Oct 2009]. 